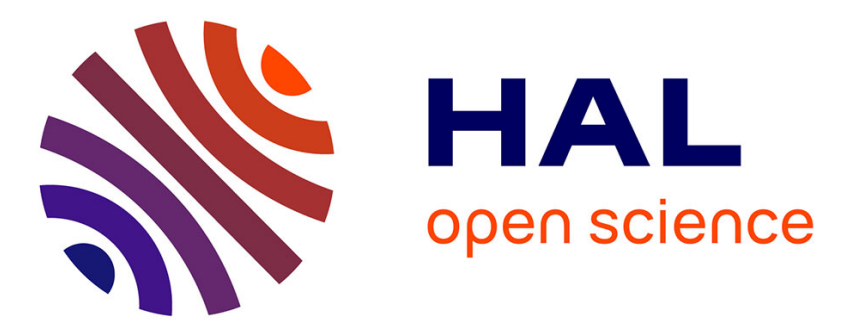

\title{
Experimental and theoretical study of density fluctuations near the stack ends of a thermoacoustic prime mover
}

Lijia Gong, Guillaume Penelet, Pascal Picart

\section{- To cite this version:}

Lijia Gong, Guillaume Penelet, Pascal Picart. Experimental and theoretical study of density fluctuations near the stack ends of a thermoacoustic prime mover. International Journal of Heat and Mass Transfer, 2018, 126, Part A, pp.580-590. 10.1016/j.ijheatmasstransfer.2018.05.027 . hal-01877645

\section{HAL Id: hal-01877645 \\ https://hal-univ-lemans.archives-ouvertes.fr/hal-01877645}

Submitted on 20 Sep 2018

HAL is a multi-disciplinary open access archive for the deposit and dissemination of scientific research documents, whether they are published or not. The documents may come from teaching and research institutions in France or abroad, or from public or private research centers.
L'archive ouverte pluridisciplinaire HAL, est destinée au dépôt et à la diffusion de documents scientifiques de niveau recherche, publiés ou non, émanant des établissements d'enseignement et de recherche français ou étrangers, des laboratoires publics ou privés. 


\title{
Experimental and theoretical study of density fluctuations near the stack ends of a thermoacoustic prime mover
}

\author{
Lijia GONG ${ }^{a, *}$, Guillaume PENELET ${ }^{\mathrm{b}}$, Pascal PICART ${ }^{\mathrm{a}, \mathrm{b}}$ \\ ${ }^{a}$ Laboratoire d'Acoustique de l'Université du Maine, LAUM UMR CNRS 6613, Le Mans Université, Avenue Olivier \\ Messiaen, 72085 Le Mans Cedex 9, France \\ ${ }^{b}$ École Nationale Supérieure d'Ingénieurs du Mans, Le Mans Université, Rue Aristote, 72085 Le Mans Cedex 09, France
}

\begin{abstract}
This paper proposes the experimental and theoretical study of nonlinear heat transport processes generated by large amplitude acoustic oscillations at the ends of a stack of plates in the presence of a temperature gradient. These processes are notably involved in the operation of thermoacoustic engines. The measurement method, a time-resolved and full-field digital holography interferometry technique, enables to measure the density fluctuations from the optical phase difference between two laser beams. This technique is applied to the analysis of density fluctuations in the vicinity of a stack submitted to a temperature gradient, firstly for the case of (uncontrolled) self-sustained acoustic oscillations generated spontaneously in a standing wave thermoacoustic prime mover, and secondly for the case of an assigned acoustic field whose amplitude is controlled by an external sound source. A theoretical model describing the advective heat transport by sound at the ends of the heated stack is also presented, and numerical simulations are then carried out. The comparison between experimental data and numerical simulations is provided for several stack positions, several sound pressure levels, and several amounts of heat supplied to the stack, and the results show good agreement between the experiments and the model.
\end{abstract}

Keywords: Thermoacoustics, Nonlinear Heat Transport, Digital Holography.

\section{Introduction}

Thermoacoustic engines are heat engines which involve the interaction of resonant gas oscillations with a porous medium (referred to as the "stack"), leading either to sound amplification (thermoacoustic prime mover) or to advective heat transport by sound (thermoacoustic heat pumps). This class of engines has been developed for about three decades [1] and has nowadays proved good performance [2] and potentiality for niche applications at moderate powers, notably for the recovery of waste heat [3. The design of thermoacoustic engines is usually based on the linear theory derived by Rott [4 which does not account for several nonlinear saturation processes such as acoustic streaming [5, 6, nonlinear propagation [7, as well as fluid separation [8] and/or complex heat exchange processes occuring at the ends of the stack and of the heat exchangers [9, 10, 11, 12. matveev2007analytical The latter effects are the ones investigated both experimentally and numerically in this paper.

The full-field and time-resolved digital holography interferometry technique (see Fig.11) is chosen to analyze the processes occuring near the stack ends in a thermoacoustic prime mover. This method allows measuring the acoustic density fluctuations (averaged along the line-of-sight) from the optical phase difference between a reference beam and an object beam passing through the acoustic resonator. Previous works by some of the authors [13, has already shown that this experimental technique is adequate for measuring harmonic and mean (time-averaged) components of density fluctuations in the vicinity of the stack, which

\footnotetext{
*. Corresponding author

Email address: Lijia.gong.etu@univ-lemans.fr (Lijia GONG)
} 


\begin{tabular}{|c|c|c|c|}
\hline \multicolumn{4}{|c|}{ Nomenclature } \\
\hline$c_{\infty}$ & speed of sound at room temperature & $T^{\prime}$ & temperature fluctuations of the fluid \\
\hline$C_{p}$ & isobaric heat capacity of the fluid $\left(C_{p}=\right.$ & $T_{0}$ & mean temperature of the fluid \\
\hline & $\left.1003 \mathrm{~J} . \mathrm{kg}^{-1} \cdot \mathrm{K}^{-1}\right)$ & $T_{\infty}$ & room temperature $\left(T_{\infty}=293 \mathrm{~K}\right)$ \\
\hline$d$ & $\begin{array}{l}\text { distance between the stack and the rigid } \\
\text { plug }\end{array}$ & $T_{c}$ & $\begin{array}{l}\text { characteristic amplitude of temperature } \\
\text { fluctuations }\end{array}$ \\
\hline$d_{s}$ & length of the stack & $\overline{T^{\prime}}$ & average temperature fluctuations of the \\
\hline$D_{i}$ & $\begin{array}{l}\text { inner diameter of the tube }\left(D_{i}=0.052\right. \\
\mathrm{m})\end{array}$ & & $\begin{array}{l}\text { fluid over the cross-sectional area of a } \\
\text { stack channel }\end{array}$ \\
\hline$D_{o}$ & outer diameter of the tube $\left(D_{o}=0.06 \mathrm{~m}\right)$ & $u_{c}$ & characteristic gas parcel displacement \\
\hline$f$ & frequency of acoustic oscillations & $v^{\prime}$ & acoustic velocity \\
\hline$k_{f}$ & $\begin{array}{l}\text { thermal conductivity of the fluid } \\
\text { at room temperature }\left(k_{f}=\right.\end{array}$ & $\kappa_{0}$ & $\begin{array}{l}\text { thermal diffusivity of the fluid (at room } \\
\text { temperature } \kappa_{\infty}=2.210^{-5} \mathrm{~m}^{2} \cdot \mathrm{s}^{-1} \text { ) }\end{array}$ \\
\hline & $\left.2.2610^{-2} \mathrm{~W} \cdot \mathrm{m}^{-1} \cdot \mathrm{K}^{-1}\right)$ & $\lambda$ & acoustical wavelength \\
\hline$k_{s}$ & effective thermal conductivity of the stack & $\omega$ & angular frequency, $\mathrm{rad} \mathrm{s}^{-1}$ \\
\hline & $\left(\kappa_{s}=0.6\right.$ W.m $\left.-K^{-1}\right)$ & $\rho^{\prime}$ & density fluctuations of the fluid \\
\hline $\begin{array}{l}L \\
p^{\prime}\end{array}$ & $\begin{array}{l}\text { tube length }(L=0.49 \mathrm{~m}) \\
\text { acoustic pressure }\end{array}$ & $\langle\rho\rangle$ & average of density through the line-of- \\
\hline$P_{0}$ & mean pressure of the fluid $\left(P_{0}=1.01510^{5}\right.$ & & $\begin{array}{l}\text { sight } \\
\text { mean density of the fluid }\end{array}$ \\
\hline$P_{c}$ & $\begin{array}{l}\mathrm{Pa}) \\
\text { characteristic amplitude of acoustic pres- } \\
\text { sure }\end{array}$ & $\begin{array}{l}\rho_{0} \\
\rho_{\infty}\end{array}$ & $\begin{array}{l}\text { mean density of the fluid at room tempe- } \\
\text { rature }\end{array}$ \\
\hline$P_{m i c}$ & peak amplitude of acoustic pressure at & $\tau$ & dimensionless time $(\tau=\omega t)$ \\
\hline$I_{m i c}$ & position $x=d$ & $\tau_{R}$ & thermal relaxation time \\
\hline$P e$ & Péclet number $\left(P e=\omega u_{c}^{2} / \kappa_{\infty}\right)$ & $\theta$ & dimensionless temperature fluctuations \\
\hline$Q_{\text {in }}$ & heat power supplied to the stack & & \\
\hline$Q_{\text {onset }}$ & $\begin{array}{l}\text { minimum of heat power to trigger self- } \\
\text { sustained oscillations }\end{array}$ & $\theta_{0}$ & $\begin{array}{l}\text { dimensionless mean temperature }\left(\theta_{0}=\right. \\
\left.\overline{T_{0}} / T_{c}\right)\end{array}$ \\
\hline$R$ & dimensionless relaxation time $\left(R=\omega \tau_{R}\right)$ & $\xi$ & dimensionless axial coordinate $\left(\xi=x / u_{c}\right)$ \\
\hline
\end{tabular}

are generated locally as a result of the abrupt transition experienced by gas parcels going back and forth through the end of the stack. These previous works were related to the description of a single experiment performed during the transient regime of wave amplitude growth in a standing wave prime mover. In this paper, additional experimental data are provided, which are obtained on the same device but for two configurations. A first configuration is the same as the one used previously [13], which means that measurements are performed during the spontaneous onset of self-sustained acoustic oscillations, while for the second configuration the acoustic field is assigned by an external sound source (so that comparison with a model is easier). Moreover, this paper presents a theoretical model describing the advective heat transport by sound at the ends of the heated stack, which is compared to experimental data.

The paper is organized as follows. In section 2 , the experimental set-up and the measurement technique are briefly described. Experiments performed during the transient regime of wave amplitude growth in a standing wave thermoacoustic prime mover are presented in section 3 A model describing the generation of higher harmonics and time-averaged components of density fluctuations at the end of the stack is presented in section 4 In section 5 additional experimental data obtained for steady-state oscillations are shown and compared with numerical simulations, while conclusions are drawn in section 6 . 


\section{Experimental set-up}

The digital holographic set-up and the thermoacoustic device are presented schematically in Fig.1(a). The thermoacoustic engine has a very simple geometry : it includes a cylindrical glass tube (with length $L$ $=49 \mathrm{~cm}$, inner diameter $D_{i}=52 \mathrm{~mm}$, outer diameter $D_{o}=60 \mathrm{~mm}$ ) opened at one end and closed by a rigid plug at the other end. Inside the tube, a porous material made up of a ceramic catalyst (the 'stack') with a length $d_{s}=48 \mathrm{~mm}$ is installed. It is structured with many square channels of semi-width $r_{s}=$ $0.45 \mathrm{~mm}$ and has the same diameter as the inner diameter of the resonator. The stack is the heart of the thermoacoustic device : it has a large surface of thermal contact with the gas, and therefore promotes the thermoacoustic amplification process which occurs within the acoustic thermal boundary layers 11. The onset of thermoacoustic oscillations is generated thanks to the existence of a temperature gradient along the stack: this temperature gradient is obtained by means of a Nichrome wire (36 cm in length, $0.25 \mathrm{~mm}$ in diameter) which is used as a heating element and is coiled through the stack end facing the rigid plug. The distribution of the temperature produced is shown schematically in Fig 1(c) : thanks to the heat leaks through lateral walls (absence of any cold heat exchanger) a temperature gradient is obtained which highest amplitude is in the vicinity of the heating wire. The self-sustained oscillations are produced when the temperature gradient reaches a critical value, which depends on the stack position along the glass tube (see ref. [14] for a marginal stability curve as a function of the stack position). The frequency of acoustic oscillations roughly corresponds to the quarter wavelength resonance, which can be calculated by $f \approx c_{\infty} / 4 L$, where $c_{\infty} \approx 343$ $\mathrm{m} / \mathrm{s}$ stands for the adiabatic sound speed evaluated at room temperature $T_{\infty} \approx 293 \mathrm{~K}$. A microphone is flush-mounted through the plugged end of the resonator to measure the frequency and amplitude of acoustic pressure oscillations.

Although the thermoacoustic device has a simple geometry, it can exhibit complicated dynamics (e.g relaxation regime of spontaneous onset/damping) which are not reproduced by numerical simulations [15] and therefore appeal for further investigations (this is one of the motivations for this study). To that purpose, the measurement of density fluctuations is performed, with the aim of getting information about local effects in heat transfer due to the abrupt transition experienced by gas parcels going back and forth through the heated stack end. A schematic representation of the set-up used to measure density fluctuations is presented in Fig 1(a) : it basically consists of an interferometer which gives a map of the optical phase difference between a reference beam and a measurement beam passing through the acoustic resonator. In the following, only the main principles of the measurement technique are described, and further information (notably about signal processing) can be found in ref. [13. The light source is a continuous red laser (optical wavelength of $660 \mathrm{~nm}$ ) which is separated into a reference beam and a probe beam by means of a polarizing cube. Both beams are then expanded and bundled to parallel rays by a collimating lens. The probe beam passes through the acoustic resonator next to the heated side of the stack, and the interference between the reference beam and the probe beam are captured by a high speed camera which is used for recording hologram sequences. The optical path length through the enlightened part of the tube may vary because of heating and/or acoustic oscillations, and this variation is captured by substracting the instantaneous optical phase difference between the two beams with the one obtained for a reference state (e.g without sound). As a result, this measurement technique gives a map of refractive index variations which itself can be related to the density map of the fluid using the Gladstone-Dale relation. Note that the measured density map is actually a map of a density averaged through the line-of-sight, denoted as $\langle\rho\rangle$ (the notation $\langle\ldots\rangle$ refers to an average along the beam path through the waveguide). The size of the map, the spatial resolution and the time resolution of measurements are determined by the performance and the settings of the high speed camera. In this study, the typical size of the observation window is of about $11.25 \times 7.6 \mathrm{~mm}^{2}$ with a pixel size of about $14.65 \mu \mathrm{m}$ and a sampling rate from 2000 up to 8000 images per second.

In the measurements described in sections 3 and 5 , two different configurations were used. The first configuration, presented in Fig. 1(a), is identical to the one used in previous works [13] : it provides measurements of the density fluctuations during a transient regime of wave amplitude growth, where the data acquisition is triggered simultaneously with the onset of the acoustic wave, during a few seconds. The results obtained with this configuration are used to analyze the impact of the position of the stack along the tube on the generation of time-averaged and higher harmonics of density fluctuations (this effect of the stack 
( a)

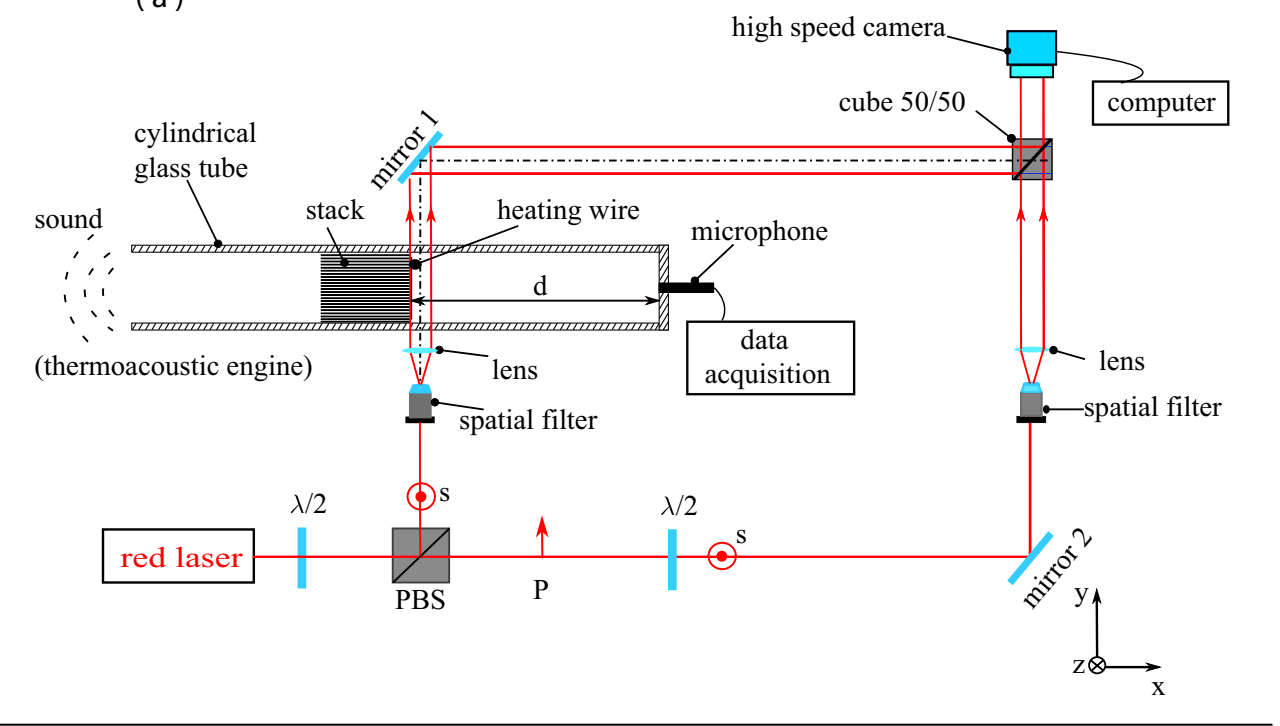

(b)

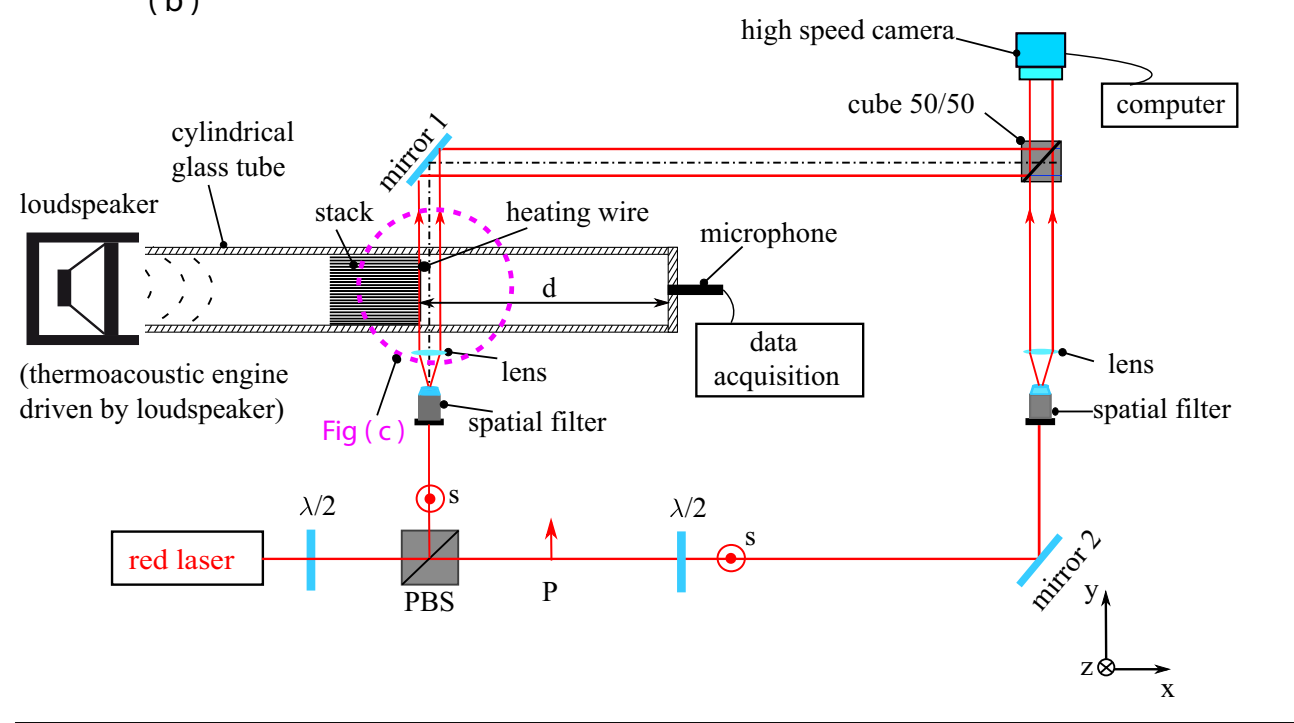

(c)

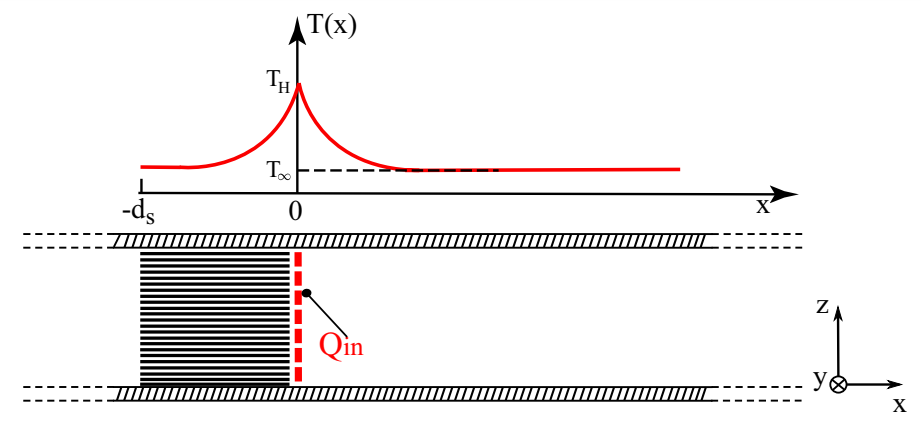

Fig. 1. Sketch of the thermoacoustic device and of the digital holographic set-up, (a) for self-sustained acoustic oscillations, (b) for assigned acoustic field (PBS : polarizing beam splitter, $s$ : sagittal polarization and $p$ : parallel polarization of laser beam.). (c) Sketch of thermoacoutic device in the vicinity of the stack where a temperature gradient is present. Note : the acoustic resonator is placed perpendicular to the gravity field. 

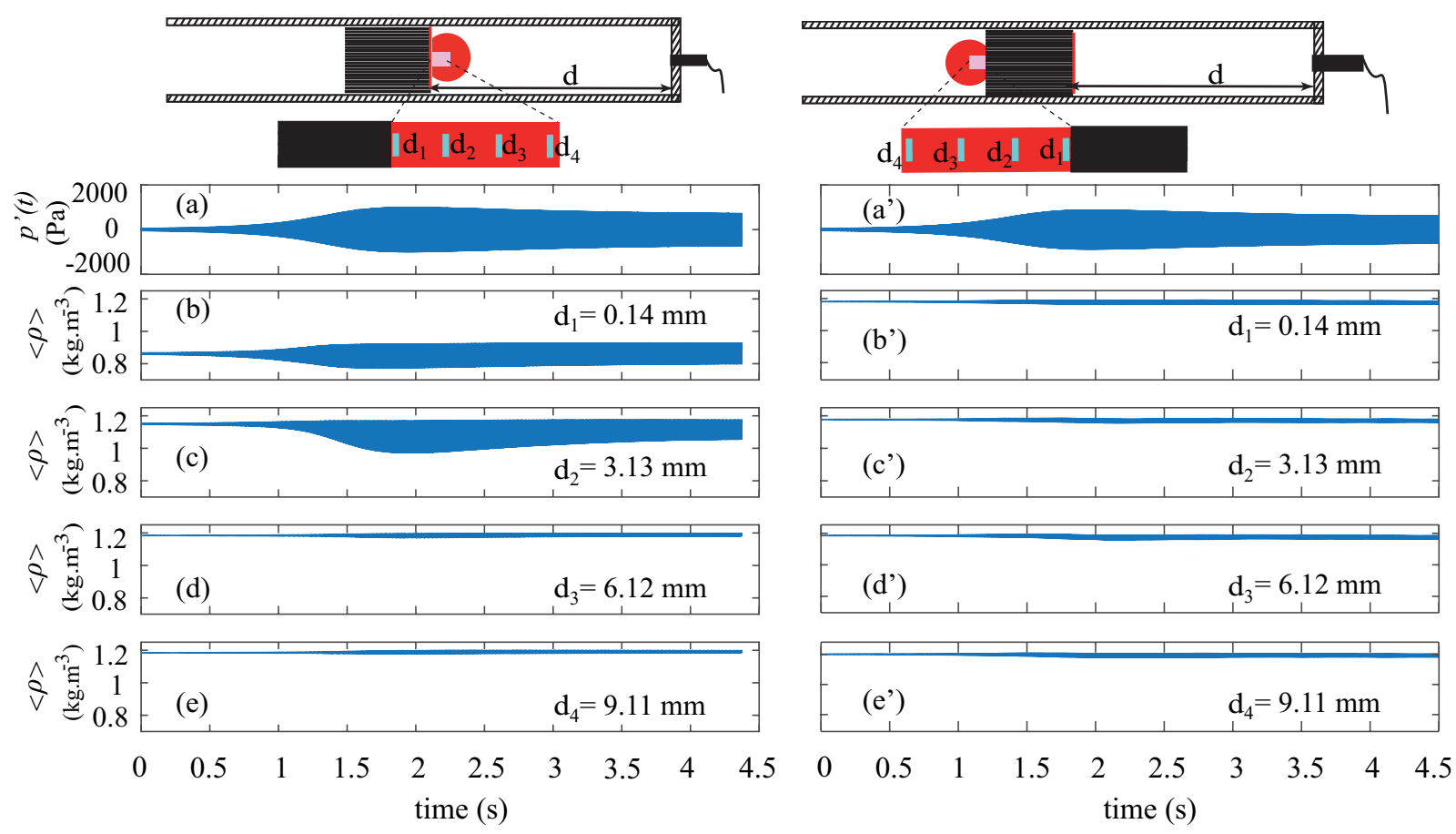

Fig. 2. Acoustic pressure (a,a') and density (b-e,b'-e') as a function of time. Density $\left(\rho=\rho_{0}+\rho^{\prime}\right)$ are presented for several distances $d_{i}$ from the stack end. The data on the left side (b-e) are obtained next to the heated side of the stack, while data on the right (b'-e') are obtained next to the non-heated side of the stack. The distance $d$ between the stack and the rigid plug is fixed to $d=18 \mathrm{~cm}$. Note : the red disc on the top of the figure corresponds to the optical beam, the pink rectangle corresponds to the camera window.

position was not investigated in previous works). However, the drawback of the method employed in this first configuration is that measurements are performed during a transient regime, so that the amplitude of acoustic oscillations is not constant during measurements. Therefore, a second configuration has also been used, which is described schematically in Fig 1(b). For this second configuration, a loudspeaker enclosure is placed very close to the open end of the thermoacoustic engine. Because of the coupling and of the additional losses caused by the presence of the loudspeaker, the onset of thermoacoustic instability does not occur (at least for the range of heat power supply employed in this study). However, this external sound source enables to generate stable acoustic oscillations (at resonance frequency) within the duct, and its amplitude is easily controlled within a range of a few tens of $\mathrm{Pa}$ up to $1 \mathrm{kPa}$. Therefore, this second configuration is well-suited for some comparisons with the model described in section 4.

\section{Experiments with self-sustained acoustic oscillations}

This section describes measurements performed when the thermoacoustic prime mover is switched on by means of external heating with the Nichrome wire, using the set-up described in Fig. 1(a). The experimental data presented in the following provide additional information to the ones presented in ref. [13] about entrance effects impacting density fluctuations next to the heated side of the stack. The measurement protocol is chosen as follows : the heat power supplied by the Nichrome wire is fixed to some value $Q_{i n}$, which is higher than the value $Q_{\text {onset }}$ corresponding to the minimum heat power required to trigger acoustic oscillations. After a few seconds, when the sound pressure measured by the microphone reaches $20 \mathrm{~Pa}$, data acquisition by the high speed camera is triggered with a sampling rate of 2000 images per second and during 4.5 seconds. Each image includes $768 \times 512$ pixels (respectively along $x$ and $z$, see Fig. 1) and a corresponding total 


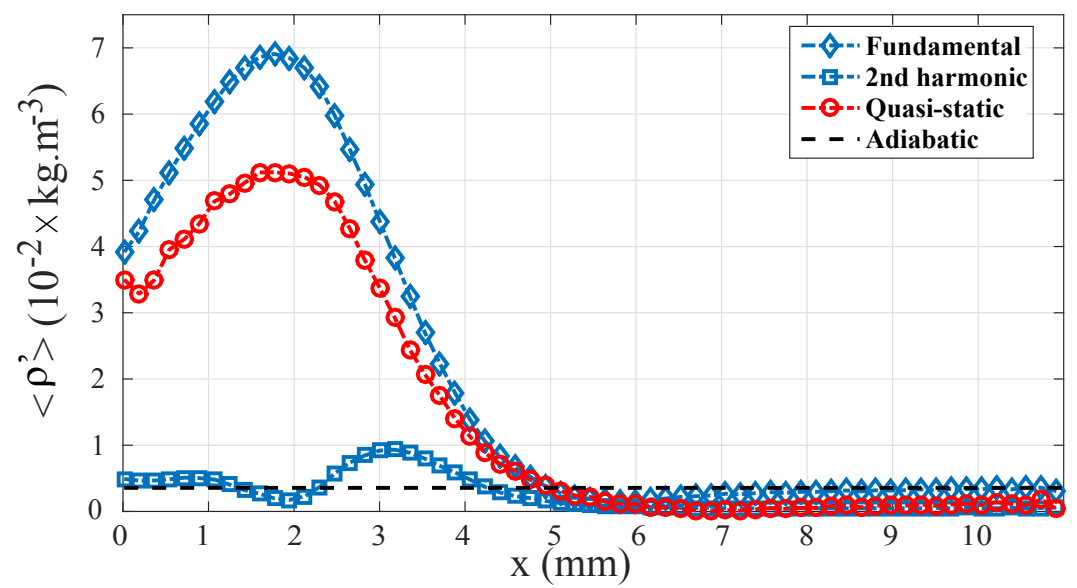

Fig. 3. Fundamental $(\diamond)$, second harmonic $(\square)$, and quasi-static module $(\circ)$ amplitudes of the density fluctuations $\left(\left\langle\rho^{\prime}\right\rangle=\right.$ $\left.\langle\rho\rangle-\left\langle\rho_{0}\right\rangle\right)$ as a function of the axial position $x(x=0$ refers to the hot stack end). The dashed horizontal black line corresponds to the adiabatic amplitude of density fluctuation estimated from the peak amplitude of the pressure measured by the microphone. Note : this quasi-static amplitude presented is without sign, it is actually negative.

length along $x$ of $11 \mathrm{~mm}$ (note that density fluctuations are homogeneous along $z$, as shown in [13]). A first image is captured at room temperature (before switching on electrical supply to the Nichrome wire) and this image is used as the reference from which fluctuations in optical path length (and therefore in density) are calculated. A first set of measurements is shown in Fig. 2 where the distance of the stack from the plugged end of the resonator is fixed to $d=18 \mathrm{~cm}$, while the heat power is fixed to $Q_{i n}=23.25 \mathrm{~W}$. Two separate experiments are performed for the configuration mentioned above, one with the probe beam crossing the resonator next to the heated side of the stack (left-side of Fig. 2), and another one with the probe beam crossing the non-heated side of the stack (right-side of Fig. 2). Figs 2 (a) and (a') present the fluctuations of acoustic pressure measured by the microphone during the two measurements, while Figs. 2(b-e) and (b'-e') present the measured (after post-processing of hologram sequences) fluctuations of density along the $x$-axis at four different distances from the stack, namely at distances $d_{1}=0.14 \mathrm{~mm}$ (b,b'), $d_{2}=3.13 \mathrm{~mm}$ (c,c'), $d_{3}=6.12 \mathrm{~mm}\left(\mathrm{~d}, \mathrm{~d}^{\prime}\right), d_{4}=9.11 \mathrm{~mm}$ (e,e'), respectively. The evolution of acoustic pressure is almost identical for these two configurations (as expected, since both the heating and the stack position are kept the same) and it shows an exponential increase followed by an overshoot (around $t \approx 1.8 \mathrm{~s}$ ) and a stabilization of the acoustic pressure amplitude around $800 \mathrm{~Pa}$ (the measured frequency of acoustic oscillations is around 171 $\mathrm{Hz}$ ). The density fluctuations measured next to the heated side of the stack are presented in Figs. 2(b-e). Oscillations of density are very small before $t \approx 0.5 \mathrm{~s}$ (because acoustic oscillations are small) and the mean value of density increases with the distance, from about 0.8 up to $1.2 \mathrm{~kg} \cdot \mathrm{m}^{-3}$ (because an axial temperature gradient is generated by the Nichrome wire). Once acoustic pressure has reached a significant amplitude (i.e. for $t \geq 0.5 \mathrm{~s}$ ), the results clearly show large oscillations of density, and the amplitude strongly depends on the observation point (as will be described with more details in Fig. 3). Moreover, the results also seem to show that the mean density strongly decreases locally, as shown especially in Fig. 2 (c) for $t \geq 1.5 \mathrm{~s}:$ this indicates that acoustic oscillations induce a mass transport phenomenon localized in the vicinity of the stack termination. The same measurements performed next to the cold side of the stack are presented in Figs. $2\left(b^{\prime}-e^{\prime}\right)$ : the results show that the amplitude of density fluctuations is much lower than in Figs. 2(b-e), and that it (almost) does not depend on the distance from the stack : the amplitude of density fluctuations is therefore just proportional to the one of acoustic pressure fluctuations (which is almost constant along the short region of analysis, much shorter than the acoustic wavelength).

The data of Fig. 2 are analyzed further in the following, and the spectral content of density fluctuations is presented in Fig. 3 as a function of the position relative to the stack termination. The blue dotted lines with 

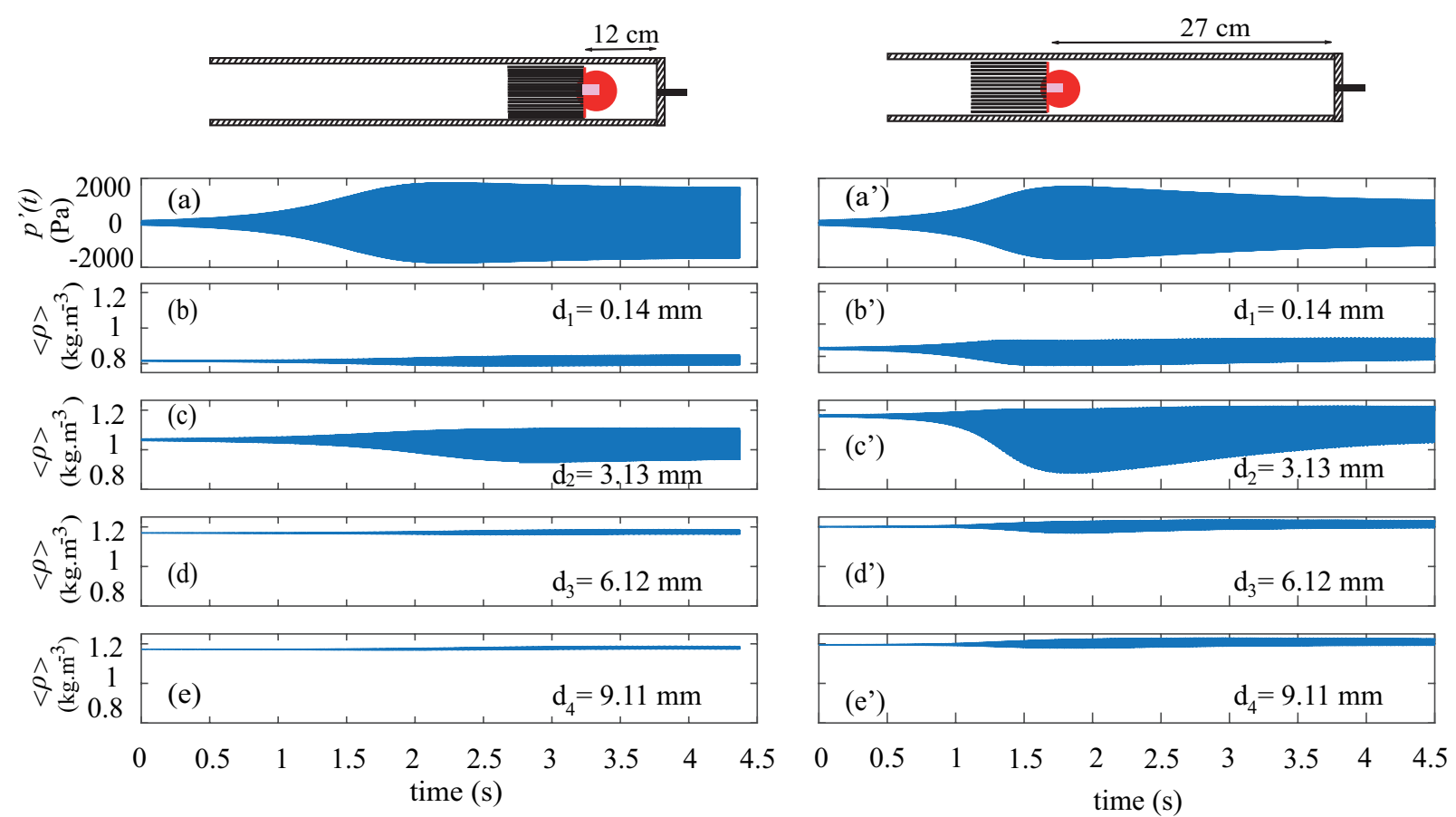

Fig. 4. Acoustic pressure (a,a') and density (b-e,b'-e') as a function of time. Density $\left(\rho=\rho_{0}+\rho^{\prime}\right)$ are presented for several distances $d_{i}$ from the hot stack end. The data on the left side (b-e) are obtained when the distance between the stack and the rigid plug equals $d=12 \mathrm{~cm}$, while data on the right (b'-e') are obtained when $d=27 \mathrm{~cm}$.

diamonds and with squares represent the amplitudes of the fundamental and the second harmonic (both are obtained from the Fourier transform of the total signal during 4.5 seconds), respectively. The red dotted line with circles represent the quasi-static amplitude of the density fluctuations as a function of the position. This amplitude is calculated from the difference between the time-average of the density during 4.5 seconds and the initial mean density at time $t=0 \mathrm{~s}$ (actually a time average of $\rho$ over the twenty first periods of acoustic oscillations). Finally, the dashed horizontal black line in Fig. 3 represents another amplitude of interest, referred to as the adiabatic amplitude, and it corresponds to the amplitude of density oscillations that one can evaluate from the measured peak amplitude $P_{m i c}$ of the pressure measured by the microphone (averaged along the total duration of the signal). If one consider that the portion of tube from the stack to the plugged end is homogeneous at room temperature. This amplitude is defined as $\rho_{a d}=P_{m i c} \cos \left(2 \pi f d / c_{\infty}\right) / c_{\infty}^{2}$. The results depicted in Fig. 3 show that the fundamental component of density fluctuations reaches maximum at a distance of about $1.8 \mathrm{~mm}$ from the stack end : this distance has the same order of magnitude than the amplitude of a gas parcel displacement $u \approx P_{m i c} \sin \left(2 \pi f d / c_{\infty}\right) /\left(2 \pi f \rho_{\infty} c_{\infty}\right) \approx 1.1$ mm evaluated next to the stack end (where $\rho_{\infty} \approx 1.2 \mathrm{~kg} \cdot \mathrm{m}^{-3}$ is the mean density of the fluid at room temperature). The results also show that far from the stack end (namely at a distance of about 10u), the amplitude of density fluctuations tends towards the adiabatic amplitude (which is more than ten times lower than the maximum value). Moreover, the results of Fig. 3 clearly show that both the second harmonic and a quasi-static component of density fluctuations are generated in the vicinity of the stack end : there is therefore a large distorsion caused by entrance effects, which however disappears far from the stack (both the second harmonic and the quasi-static component tend towards zero as $x$ increases). All of these observations are consistent with the ones presented in ref. [13, but an additional conclusion which comes from the comparative analysis of Figs. 2 (b-e) and 2(b'-e') is that the presence of a temperature gradient has a large impact on both the amplitude and the spectral content of density fluctuations next to the stack.

In Fig. 4, the impact of the position of the stack along the tube is analyzed. The left-side of Fig. 4 corresponds to the results obtained when the stack is placed at a distance $d=12 \mathrm{~cm}$ from the rigid plug, 
while the right-side of Fig. 4 presents the results obtained when $d=27 \mathrm{~cm}$. As mentioned earlier, it is well-known that the position of the stack along the tube impacts the threshold of acoustic oscillations : this is because the thermoacoustic amplification process is controlled by both the relative amplitude and the phasing of pressure and velocity oscillations (which are not constant along the tube). Therefore, the value of $Q_{\text {onset }}$ depends on $d$. Thus $Q_{\text {in }}$ is fixed to $29.24 \mathrm{~W}$ for the configuration with $d=27 \mathrm{~cm}$, while $Q_{i n}$ is fixed to $23.25 \mathrm{~W}$ for $d=12 \mathrm{~cm}$. From the comparative analysis of the dynamics of the acoustic pressure evolution, which is presented for both configurations in Figs 4 (a) and (a'), we notably see that the position of the stack impacts the peak amplitude amplitude of acoustic pressure oscillations which reaches $p \approx 800 \mathrm{~Pa}$ for $d=12 \mathrm{~cm}$, while it is significantly lower (around $500 \mathrm{~Pa}$ ) for $d=27 \mathrm{~cm}$. However, as shown in Figs 4 (b-e) and (b'-e'), the amplitude of density fluctuations at several distances from the hot stack end are significantly larger when $d=27 \mathrm{~cm}$ than when $d=12 \mathrm{~cm}$. Therefore, although the acoustic energy stored in the resonator is larger when the stack is placed closer to the plugged end, the distortion of density fluctuations in the vicinity of the stack increases as the stack is moved away from the plug. It is also worth pointing out that the overshoot process observed for both pressure and density fluctuations is more pronounced when the stack is placed far from the plug than when $d=12 \mathrm{~cm}$. These results indicate that the ratio of velocity to pressure amplitudes at the position of the stack is an important parameter which controls the local magnitude of density fluctuations. In the next section, a model is presented, which aims at reproducing the processes observed in experiments.

\section{Description of temperature distorsion close to the stack termination}

As already pointed out in previous research works [16, 17, 8, 18, 9, the linear thermoacoustic theory fails in describing heat and mass transport processes near the stack terminations, because the abrupt transition experienced by gas parcels oscillating back and forth through the stack ends leads to significant local nonlinearities. These effects have two origins : one is related to the geometrical singularity at the end of the stack which leads to flow separation and viscous dissipation [8, 18, while the other one is related to a singularity in terms of heat transfer [16, 17] which leads to the distorsion of temperature fluctuations [9, 13]. In this section, we provide a simplified description of the latter effect, which is mainly based on the assumptions, (1) that the fluid is inviscid, (2) that the stack plates are infinitely thin and have an infinite thermal conductivity, and (3) that the transverse heat conduction inside the stack is taken into account by means of a relaxation time approximation. The model presented in the following is actually an extension of the one proposed by Gusev et al. [17, and it includes some effects due to axial heat conduction and the presence of a temperature gradient. This model will be used to predict the generation of higher harmonics and timeaveraged components of temperature fluctuations, which themselves will be related to the fluctuations of density.

The geometry of the considered device is the one of Fig. 1 (c), and it is assumed that the gas experiences acoustic oscillations along $x$ (the origin $x=0$ is fixed at the hot side of the stack). If the fluid is assumed to be inviscid, and if the thermal conductivity of the fluid is assumed independant of the temperature, the equation describing the temperature deviation $T^{\prime}$ of the temperature from its mean (non-oscillating) value $T_{0}(x)$ is given by [17, 19

$$
\partial_{t} T^{\prime}+v^{\prime} \partial_{x}\left(T_{0}+T^{\prime}\right)=\frac{\partial_{t} p^{\prime}+v^{\prime} \partial_{x} p^{\prime}}{\rho_{0} C_{p}}+\kappa_{0}\left(\partial_{x x}^{2}+\nabla_{\tau}^{2}\right) T^{\prime},
$$

where $p^{\prime}$ denotes the pressure fluctuations around the static pressure $P_{0}, \rho_{0}$ stands for the mean density of the fluid, $C_{p}$ is the isobaric heat capacity of the fluid, $\kappa_{0}$ is the thermal diffusivity of the fluid, and where $\nabla_{\tau}^{2}$ denotes the transverse part of the Laplace operator $\left(\partial_{x}\right.$ and $\partial_{t}$ stand for $x$ and time derivatives, respectively). As far as the axial temperature gradient is large only in the vicinity of the stack end within a region which is much smaller than the acoustic wavelength $\lambda$, the spatial distribution of pressure fluctuations in the region extending from $x=0$ to the rigid end $x=d$ of the acoustic resonator is not strongly affected by the temperature gradient [20, and it can therefore be written as $p^{\prime} \approx P_{m i c} \cos [2 \pi(x-d) / \lambda] \cos (\omega t)$, where $P_{m i c}$ is the 
peak amplitude of acoustic pressure at position $x=d$ (the plugged end of the resonator). The same argument holds for the acoustic velocity which is approximated by $v^{\prime} \approx\left(2 \pi P_{m i c} / \rho_{\infty} \lambda \omega\right) \sin [2 \pi(x-d) / \lambda] \sin (\omega t)$, where $\rho_{\infty}$ refers to the density of the fluid at room temperature $T_{\infty}$. Eq. (1) is simplified in the following to describe local distorsion of the temperature fluctuations around $x=0$, within a compact region extending up to a few gas parcels displacements. Here the word compact means that this region is much smaller than the acoustic wavelength, so that the pressure and axial velocity are almost independant on $x$ within this region, and therefore the two following approximations

$$
p^{\prime} \approx P_{m i c} \cos \left(\frac{2 \pi d}{\lambda}\right) \cos (\omega t)=P_{c} \cos (\omega t)
$$

and

$$
v^{\prime} \approx-\frac{2 \pi P_{m i c}}{\lambda \rho_{\infty} \omega} \sin \left(\frac{2 \pi d}{\lambda}\right) \sin (\omega t)=\omega u_{c} \sin (\omega t)
$$

can be reported in Eq. (1). Moreover, the term $v^{\prime} \partial_{x} p^{\prime}$ on the right hand side of Eq. (1) can be neglected in comparison with $\partial_{t} p^{\prime}$, because an estimate leads to $v^{\prime} \partial_{x} p^{\prime} \sim\left(v^{\prime} / \lambda\right) p^{\prime}$ with $\left(v^{\prime} / \lambda\right) \ll 1$. The simplifications mentioned above are the same as those formulated by Gusev in ref. [17] but an additional term $v^{\prime} \partial_{x} T_{0}$ is present in Eq. (1) which describes the effect of an axial temperature gradient on the acoustic temperature fluctuations. The term $\kappa_{0} \partial_{x x}^{2} T^{\prime}$ representing the dynamic effect of axial heat diffusion was neglected in ref. 17. as it was considered as small in comparison with the hydrodynamic temperature transport (described by the term $v^{\prime} \partial_{x} T^{\prime}$ ) but Berson et al. 9. have shown that it may play a role for large amplitude of acoustic oscillations. Therefore we keep this term in Eq. (1), but for the sake of simplicity we neglect the dependance on $x$ of the thermal diffusivity $\kappa_{0}$ (due to temperature variations) which is replaced by its value $\kappa_{\infty}$ evaluated at room temperature $T_{\infty}$. We also neglect the dependance of $\rho_{0}$ with the axial coordinate in the term $\partial_{t} p^{\prime} /\left(\rho_{0} C_{p}\right)$, which is replaced by $\partial_{t} p^{\prime} /\left(\rho_{\infty} C_{p}\right)$, where $\rho_{\infty}$ is the mean density evaluated at room temperature. Therefore, all approximations mentioned above lead to the equation :

$$
\partial_{t} T^{\prime}+v^{\prime} \partial_{x}\left(T_{0}+T^{\prime}\right)=\frac{\partial_{t} p^{\prime}}{\rho_{\infty} C_{p}}+\kappa_{\infty}\left(\partial_{x x}^{2}+\nabla_{\tau}^{2}\right) T^{\prime},
$$

Following Gusev et al. [17, this equation is integrated over a cross-section $S$ of a pore in the stack, and the relaxation-time approximation is formulated to simplify the last term on the right-hand side. This approximation amounts to describe the lateral heat transport between the fluid and the stack walls by means of a Newton's law of cooling, i.e. to assume that this lateral heat flux is proportional to the difference between the temperature of the solid, $T_{0}$, and that of the fluid averaged along a cross-section of a pore, $T_{0}+\overline{T^{\prime}}$. As a result, Eq. (4) is re-written as

$$
\partial_{t} \overline{T^{\prime}}+v^{\prime} \partial_{x} T_{0}+v^{\prime} \partial_{x} \overline{T^{\prime}}=\frac{\partial_{t} p^{\prime}}{\rho_{\infty} C_{p}}+\kappa_{\infty} \partial_{x x}^{2} \overline{T^{\prime}}-\frac{\overline{T^{\prime}}}{\tau_{R}},
$$

where the relaxation time $\tau_{R}$ has been introduced (see ref. [17 for more details). This parameter is a phenomenological parameter which does not account for all the complexity of heat transfer processes occuring at the stack ends, but it can be used to describe the abrupt transition experienced by gas parcels which cross the stack ends : in the following, the parameter $\tau_{R}$ will have a finite value for gas parcels inside the stack $(x<0)$ which exchange heat with lateral walls, while $\tau_{R}$ will tend towards infinity to describe the adiabatic temperature oscillations of gas parcels outside the stack $(x>0)$.

Finally, the last step of this analytical development consists in re-writing Eq. (5) in a dimensionless form. To that purpose, we introduce the characteristic amplitude of temperature fluctuations $T_{c}$ defined as $T_{c}=-P_{c} /\left(\rho_{\infty} C_{p}\right)$, and we also define the dimensionless variables $\tau=\omega t$ and $\xi=x / u_{c}$ (where the length $u_{c}$ introduced in Eq. (3) denotes the amplitude of gas parcel displacement at position $x=0$ ) so that Eq. (5) is transformed into :

$$
\frac{\partial \theta}{\partial \tau}+\sin \tau\left(\frac{\partial \theta_{0}}{\partial \xi}+\frac{\partial \theta}{\partial \xi}\right)=\sin \tau-\frac{\theta}{\mathrm{R}}+\frac{1}{\mathrm{Pe}} \frac{\partial^{2} \theta}{\partial \xi^{2}}
$$


where $R=\omega \tau_{R}, P e=\omega u_{c}^{2} / \kappa_{\infty}$ and $\theta_{0}=\frac{T_{0}}{T_{c}}$. This equation describes the fluctuation of temperature locally at the stack-fluid interface and aims at reproducing, at least qualitatively, the generation of temperature harmonics as well as the generation of a mean component on temperature, due to the non-linearity of the heat exchanges at the end of the stack of plates. The key parameter of this equation results from the relaxationtime approximation, and is represented by the phenomenological parameter $R=\omega \tau_{R}$, which is here used to account for the abrupt transition in the thermal coupling between the oscillating gas and the stack walls, without going into the detail of the stack geometry. In the following, we will consider that inside the stack $(\xi \leq 0)$ one has $R=1$ while outside the stack $(\xi>0)$ it tends towards infinity : this assumption amounts to considering that the typical size of a stack pore is such that the thermoacoustic process is optimum $(R \approx 1)$ while the process is purely adiabatic outside the stack $(R \gg 1)$. In the following, Eq. (6) will be solved numerically using a finite-difference scheme. This requires to know the mean temperature gradient $T_{0}(x)$, which will be evaluated from the experimental data. The resulting solution for the temperature fluctuations $\overline{T^{\prime}}$ around the stack ends will be then transformed into density fluctuation $\overline{\rho^{\prime}}$ using the formula

$$
\frac{\overline{\rho^{\prime}}(x, t)}{\rho_{0}(x)}=\frac{P_{c}}{P_{0}} \cos (\omega t)-\frac{\overline{T^{\prime}}(x, t)}{T_{0}(x)}
$$

stating that the gas is an ideal gas experiencing small perturbations of temperature and pressure.

\section{Experiments with an assigned acoustic field and comparison with theory}

This section presents experimental data compared with numerical simulations. The experimental set-up is the one described in Fig. 1(b) : contrarily to the results presented in section 3 the acoustic field is sustained by means of an external sound source, which enables to control much more easily a stable amplitude of acoustic oscillations during measurements. The measurements are performed at different acoustic pressure levels $\left(P_{\text {mic }}=300,600\right.$ and $\left.870 \mathrm{~Pa}\right)$ with the frequency $f=171 \mathrm{~Hz}$ (the same as the one of spontaneous oscillations), at different heat power levels $\left(Q_{i n}=15.4,18.9 \mathrm{~W}\right)$ and at different positions of the stack along the tube $(d=18,23$ and $27 \mathrm{~cm})$.

The measurement results are compared with theoretical data,which are obtained by solving Eq. (6) numerically. A finite-difference scheme is used for both time and space, and the computational domain ranges from $\xi=-10$ (or $x=-10 u$ ) up to $\xi=30$, while the calculations are performed over a total time of $\tau=6000 \pi$ (i.e more than 3000 acoustic periods) to make sure that convergence and steady-state solutions have been reached for all components of temperature fluctuations. Prior to numerical solving, some unknown parameters have to be determined from experimental data. For each set of measurements, the amplitude dependant parameters $P_{c}=P_{m i c} \cos [(2 \pi d)(\lambda)], u_{c}=-\left(2 \pi P_{m i c}\right) /\left(\lambda \rho_{\infty} \omega^{2}\right) \sin [(2 \pi d) /(\lambda)], P e=$ $\omega u_{c}^{2} / \kappa_{\infty}$, and $T_{c}=-P_{c} /\left(\rho_{\infty} C_{p}\right)$ are estimated from the assigned amplitude $P_{m i c}$ of acoustic oscillations and the distance $d$ between the stack and the rigid plug. The temperature distribution $T_{0}(x)$, which notably depends on $Q_{i n}$, also needs to be estimated from experiments. To that purpose, a first measurement of the mean density $\rho_{0}(x)$ is performed once heat supply has been switched on and steady-state temperature is reached, but before switching on the loudspeaker. This measurement of $\rho_{0}(x)$ enables to calculate the mean temperature distribution $T_{0}(x)$ along the spatial domain outside the stack (i.e. for $x \geq 0$ ) by making use of the ideal gas law. The temperature distribution inside the stack (for $x \leq 0$ ), however, cannot be directly obtained from experiments and has to be evaluated indirectly. As the computational domain inside the stack ranges up to 10 times the gas parcel displacement, it is assumed that the resulting length is sufficiently short so that the temperature distribution is assumed locally linear inside the stack. For the set of measurements described below, the maximum amplitude of gas parcel displacement (estimated for $d=27 \mathrm{~cm}$ and $P_{m i c}=870$ $\mathrm{Pa}$ ) is around $\left|u_{c}\right| \approx 1.5 \mathrm{~mm}$, so that for this most unfavorable situation, it is assumed that the temperature distribution is linear along the stack over a length of about $15 \mathrm{~mm}$. Thanks to this approximation, the slope of the temperature distribution in the stack $(x \leq 0)$ is evaluated by the following formula :

$$
\left.k_{s} S \partial_{x} T_{0}\right|_{x=0^{-}}=\left.k_{f} S \partial_{x} T_{0}\right|_{x=0^{+}}+Q_{i n}
$$



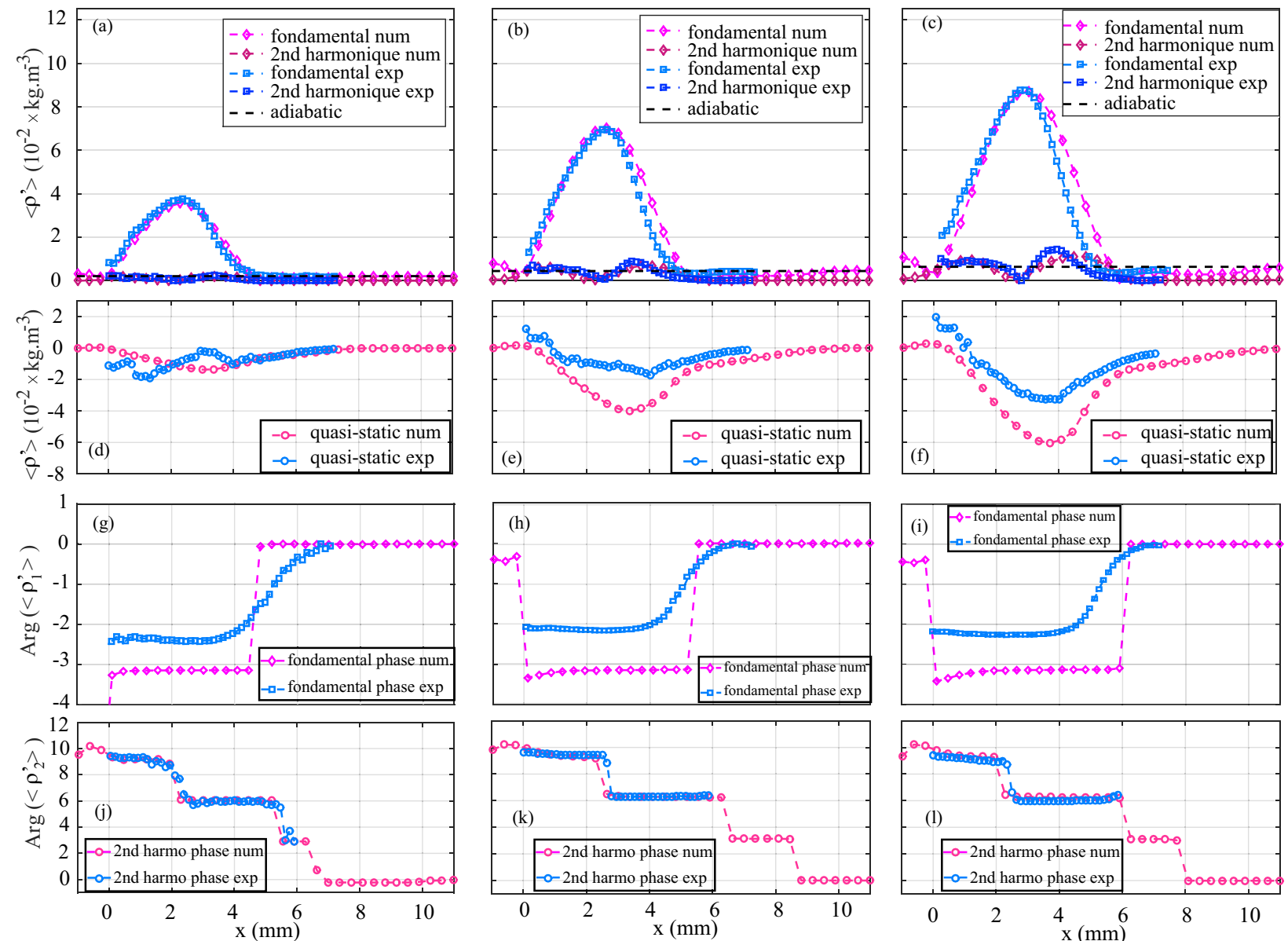

Fig. 5. (a), (b) and (c) : Comparison between the numerical and experimental results (amplitude of fundamental and second harmonic components of density) as a function of $x$, under conditions $d=18 \mathrm{~cm}, Q_{i n}=15.4 \mathrm{~W}$ and for different amplitudes of forcing, namely $P_{m i c}=300 \mathrm{~Pa}(\mathrm{a}), P_{m i c}=600 \mathrm{~Pa}$ (b), and $P_{m i c}=870 \mathrm{~Pa}$ (c). (d), (e) and (f) : Comparison between the numerical and experimental results for the quasi-static component of density fluctuations, under the same conditions, namely $P_{m i c}=300 \mathrm{~Pa}(\mathrm{~d}), P_{m i c}=600 \mathrm{~Pa}(\mathrm{e})$, and $P_{m i c}=870 \mathrm{~Pa}(\mathrm{f}) .(\mathrm{g}),(\mathbf{h}),(\mathrm{i})$ and (j), (k), (l) : Phase of the fondamental (g-i) and the second harmonic $(\mathrm{j}-\mathrm{k})$ components of the density fluctuations $\rho^{\prime}$, under the same conditions. This phase is calculated with reference to the phase of density fluctuations far from the stack.

where $k_{f}=2.26 \times 10^{-2} \mathrm{~W} / \mathrm{m} / \mathrm{K}$ stands for the thermal conductivity of the fluid, $k_{s}$ stands for the effective thermal conductivity of the stack, and $S=\pi\left(D_{i} / 2\right)^{2}$ denotes the cross-sectional area of the duct. In accordance with previous works [15, $k_{s}$ is evaluated to $k_{s} \approx 0.6 \mathrm{~W} / \mathrm{m} / \mathrm{K}$. Finally, the dimensionless relaxation time $R=\omega \tau_{R}$ also has to be fixed : the formulated choice is identical to the one made by Berson et al. [9], and the values of $R=1$ for $x \leq 0$ and $R=10^{4}$ for $x>0$ are retained. This is obviously an arbitrary choice as the value of $R$ depends on the size and the shape of the stack channels, but the physical justification is that the value of $R=1$ corresponds to an optimal regime of thermoacoustic transport, while the large value $R=10^{4}$ has the meaning of an adiabatic regime.

The comparison of the simulation results and the experimental results for a stack position $d=18 \mathrm{~cm}$, a heat power supply $Q_{i n}=15.4 \mathrm{~W}$ and three levels of sound pressure are presented in Fig. 5 . Comparison is performed for the fundamental and the second harmonic components of density fluctuations as a function of the position $x$ (upper figures), but also for the quasi-static component of density fluctuations. The results show good agreement between experiments and theory for both the fundamental and the second harmonic components of density fluctuations, especially at low amplitudes. At larger amplitudes some differences are observed, but the agreement can be considered as good when considering that the numerical model was 
derived from several aproximations. The comparison of experiments with theory concerning the latter component, i.e. the quasi-static amplitude of density oscillations, appeals for further comments because this comparison is actually not straightforward. The theoretical quasi-static component of density fluctuations refers to the time-average (over 40 acoustic periods) of the steady-state numerical solution, while the experimental quasi-static component is obtained from the difference between the measured time-average of $\left\langle\rho^{\prime}\right\rangle$ (with sound) and the mean density $\rho_{0}(x)$ measured before sound is switched on. Due to this, the quasi-static component of density fluctuations obtained experimentally is not only generated by the non-linearities in heat transfer considered by the model, but also by the mean thermoacoustic transport of heat inside the stack (i.e. advective heat transport by sound described by the linear thermoacoustic theory (i.e. advective heat transport by sound described by the linear thermoacoustic theory 1, 4). This consideration may possibly explain why the results presented in Fig 5 show a significant difference between experiments and theory around the stack end $(x \approx 0)$. It is however worth noting that far from the stack end (where thermoacoustic streaming is absent), there is a good qualitative agreement between experiments and theory, especially at high amplitudes of acoustic oscillations where both curves are very close. The associated phases of the fundamental $(\mathrm{g}, \mathrm{h}, \mathrm{i})$ and second harmonic $(\mathrm{j}, \mathrm{k}, \mathrm{l})$ components of density fluctations obtained both from the experiments and the model are also presented in Fig. 5. These phases are plotted as a function of the axial position $x$, using as the reference phase the one of density oscillations far from the stack. From the comparison of our experimental data with the results of the model, we can see that although the agreement is not perfect, the main trends observed in experiments are reproduced by the model. In particular, a phase jump is clearly observed for both experiments and theory in the fundamental components of $\left\langle\rho^{\prime}\right\rangle$, see Fig. 5 (g,h,i) around $x \approx 5 \mathrm{~mm}$. This phase jump can be explained by the fact that, as described below, the density fluctuations result from a linear combination of pressure and temperature fluctuations. The presence of a steep axial temperature gradient leads to an increase of the amplitudes of temperature fluctuations (whose phase remains close to the one of the acoustic pressure almost everywhere, according to our model) and therefore , as stated in Eq (7), this leads to a competition between both contributors of density fluctuations, namely $P_{c} \cos (\omega t)$ and $-\overline{T^{\prime}}$. That is the reason why the model predicts a change in sign of density fluctuations (or equivalently a phase jump of $\pi$ ) once the contribution of $\overline{T^{\prime}}$ counteracts the contribution of $P_{c} \cos (\omega t)$. This phase jump is also observed in experiments and at the same position as the one predicted by the model, but its magnitude is lower (around $2 \mathrm{rad}$.) : this might be explained by the fact that we measure a density averaged through the line of sight while the temperature distribution is not uniform through the duct cross section. The Phase of the second harmonic components are very closed between experiment data and the result of our mode when the axial position $x<6 \mathrm{~mm}(\mathrm{i}, \mathrm{j}, \mathrm{k})$. Actually, we did not plot the second harmonic components from the experiments at $x>6 \mathrm{~mm}$, because far from the stack, the amplitude of the second harmonic exponent is so small that it's phase is not significant. More generally, the information about the phase between acoustic variables is an important information, because the advective heat transport induced by acoustic oscillations through the stack ends involves the time-average between the temperature and the velocity fluctuations.

The same kind of comparison between experiments and theory is presented in Fig. 6, where the heat power supply has been increased up to $Q_{i n}=18.9 \mathrm{~W}$. The conclusion drawn from the analysis of Fig. 6 6 are similar to the ones drawn from Fig. 5: the numerical model succeeds in reproducing the amplitudes of the fundamental and second harmonic components of density fluctuations observed in experiments, especially at moderate sound pressure levels, while some important discrepancies are observed for the quasi-static component. For the latter component, the qualitative agreement is however good, and the order of magnitude of the generated mean component of density is the same for both experiments and theory. From the comparative analysis of Figs 5 and 6 , it is concluded that increasing the heat power supply $Q_{\text {in }}$ while keeping the sound pressure level constant leads to an increase of entrance effects observed in density fluctuations : this can be explained by the fact that increasing $Q_{i n}$ leads to an increase of the temperature gradient $\partial_{x} T_{0}$ which strongly impacts temperature and density fluctuations next to the stack end.

In Fig. 7, the variations of different components of the density fluctuations are presented as a function of $x$ for different sound pressure levels, with a heat power input $Q_{i n}=15.4 \mathrm{~W}$, and when the stack has been moved from $d=18$ up to $d=23 \mathrm{~cm}$. Moving the stack towards the open end of the duct leads to a decrease of the characteristic acoustic pressure amplitude $P_{c}\left(P_{m i c}\right.$ being kept the same) and an increase of 

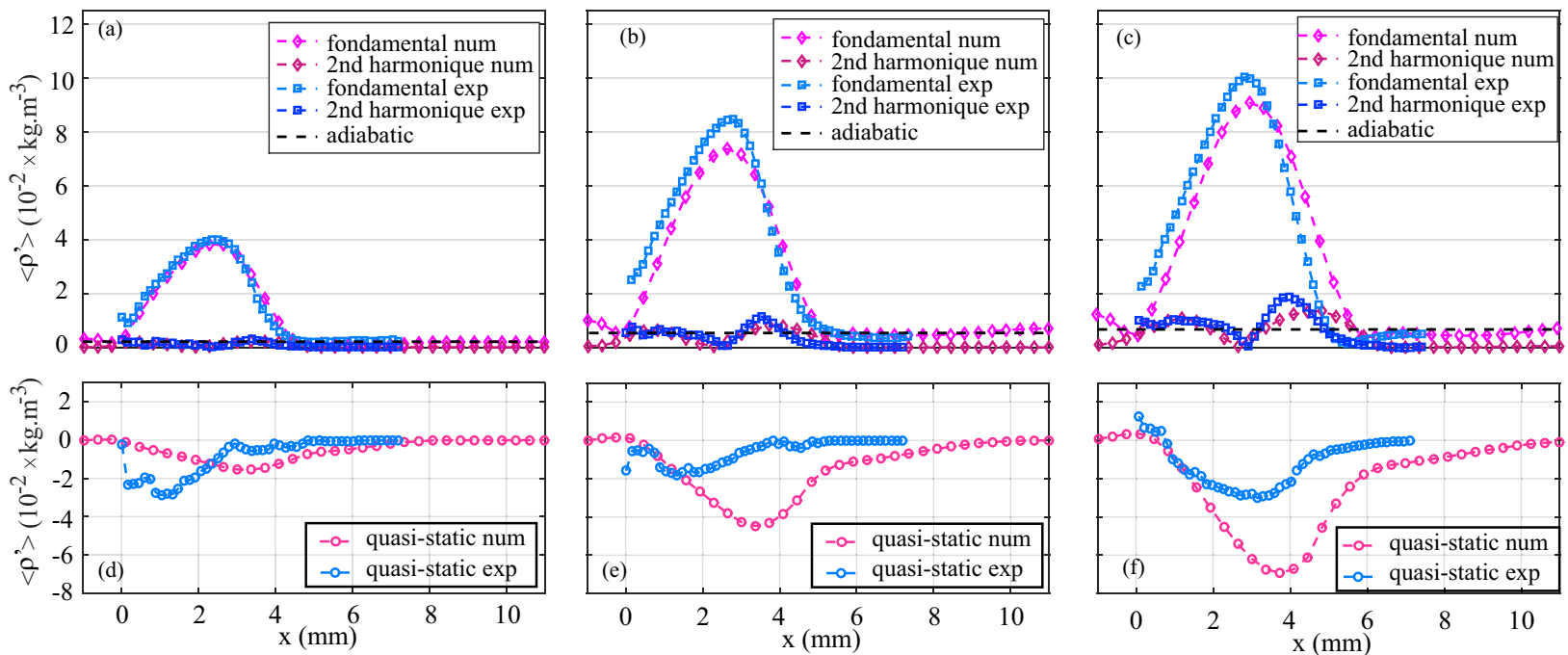

Fig. 6. (a), (b) and (c) : Comparison between the numerical and experimental results (amplitude of fundamental and second harmonic components of density) as a function of $x$, under conditions $d=18 \mathrm{~cm}, Q_{i n}=18.9 \mathrm{~W}$ and for different amplitudes of forcing, namely $P_{m i c}=300 \mathrm{~Pa}(\mathrm{a}), P_{m i c}=600 \mathrm{~Pa}(\mathrm{~b})$, and $P_{m i c}=870 \mathrm{~Pa}$ (c). (d), (e) and (f) : Comparison between the numerical and experimental results for the quasi-static component of density fluctuations, under the same conditions.
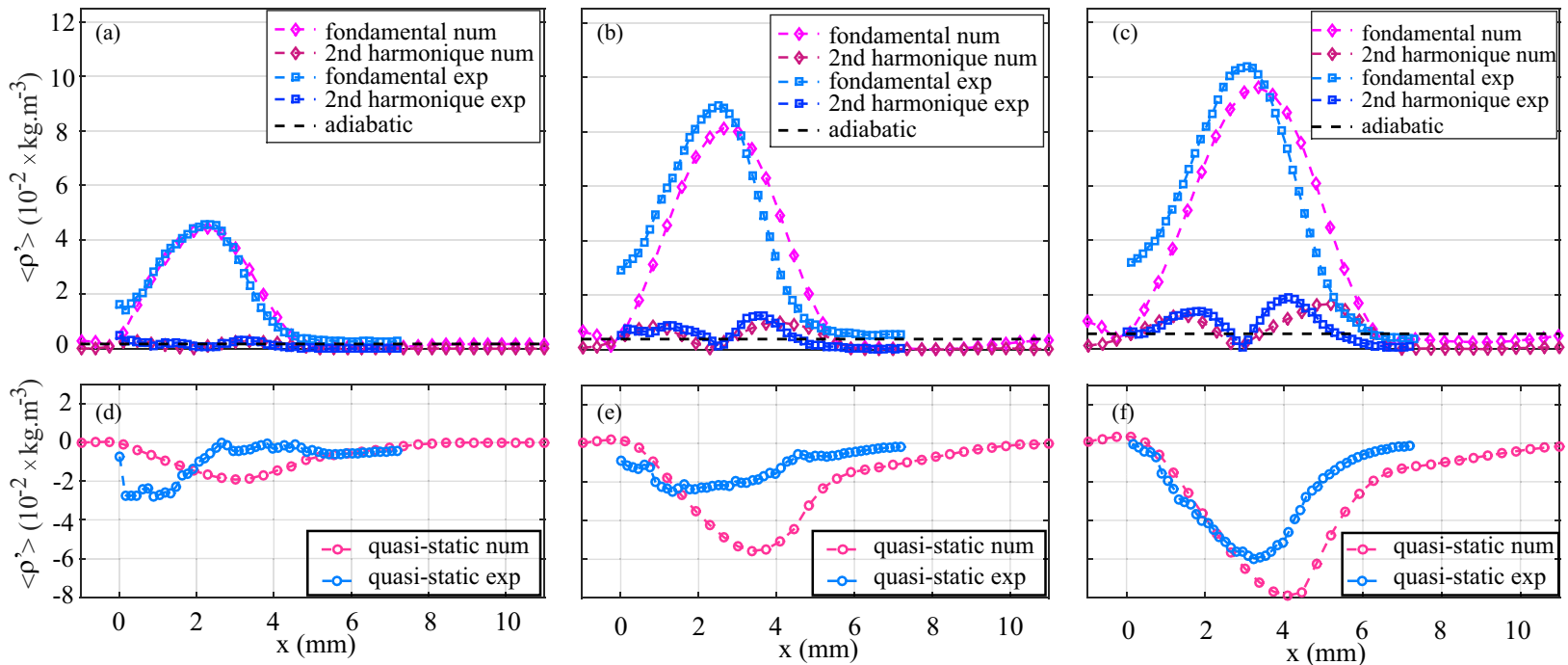

Fig. 7. (a), (b) and (c) : Comparison between the numerical and experimental results (amplitude of fundamental and second harmonic components of density) as a function of $x$, under conditions $d=23 \mathrm{~cm}, Q_{i n}=15.4 \mathrm{~W}$ and for different amplitudes of forcing, namely $P_{m i c}=300 \mathrm{~Pa}(\mathrm{a}), P_{m i c}=600 \mathrm{~Pa}(\mathrm{~b})$, and $P_{m i c}=870 \mathrm{~Pa}$ (c). (d), (e) and (f) : Comparison between the numerical and experimental results for the quasi-static component of density fluctuations, under the same conditions. 

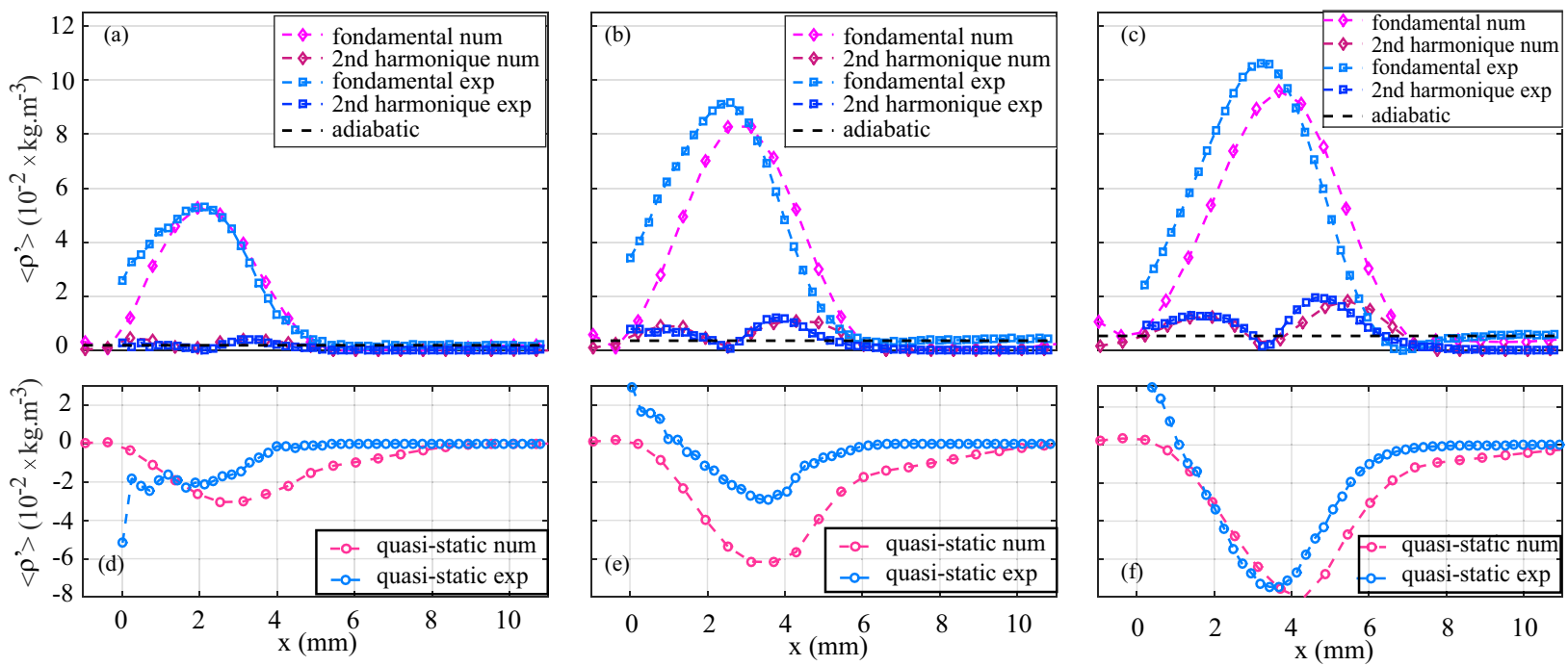

Fig. 8. (a), (b) and (c) : Comparison between the numerical and experimental results (amplitude of fundamental and second harmonic components of density) as a function of $x$, under conditions $d=27 \mathrm{~cm}, Q_{i n}=15.4 \mathrm{~W}$ and for different amplitudes of forcing, namely $P_{m i c}=300 \mathrm{~Pa}(\mathrm{a}), P_{m i c}=600 \mathrm{~Pa}(\mathrm{~b})$, and $P_{m i c}=870 \mathrm{~Pa}$ (c). (d), (e) and (f) : Comparison between the numerical and experimental results for the quasi-static component of density fluctuations, under the same conditions.

the acoustic velocity, compared to the results presented in Fig. 5 . From the comparative analysis of Figs. 7 and 5 we see that when the stack is moved away from the rigid plug (while both the heat supply and the acoustic power stored in the resonator are kept constant) the amplitude of nonlinear density fluctuations is increased, which is consistent with the observations made with spontaneous oscillations in section 3 , Moreover, the distance up to which entrance effects impact the density fluctuations is increased when $d$ is increased. It is also worth pointing out that the agreement between experiments and theory is very good at large amplitudes concerning the quasi-static component of $\langle\rho\rangle$.

Finally, the effect of moving the stack further away from the plug is analyzed in Fig. 8, where $d$ is fixed to $27 \mathrm{~cm}$ (still with $Q_{i n}=15.4 \mathrm{~W}$ ). Both experimental and theoretical data show that increasing $d$ leads to an increase of the distorsion (the amplitude of each component increases) and also with an increase of the typical distance from the stack up to which entrance effects are visible (the latter consideration is quite evident if one considers that the gas parcel displacement amplitude increases as $d$ increases). It is also interesting to note that the agreement between experiments and theory concerning the quasi-static amplitude of density is better for large values of $d$ than for small values of $d$ (in Fig 5) : this fact is somehow surprising when considering that the model does not account for aerodynamical entrance effects due to viscosity which should be more and more effective (and might impact more and more the time-averaged temperature) when the gas parcel displacement is increasing.

\section{Conclusion}

The experimental and theoretical results presented in this paper confirm that the temperature and density oscillations are highly nonlinear up to distances of the order of a gas parcel displacement from the stack edge, as already pointed out in previous studies [17, 9, 13. The main contribution of this study is to investigate the impact of an axial temperature gradient due to the heating of the stack end, which is a usual situation encountered in thermoacoustic engines. A model is introduced in this paper which mainly relies on the relaxation-time approximation applied to the motion of an inviscid gas oscillating through a temperature gradient. The model succeeds in reproducing qualitatively (and even quantitatively) the experimental results obtained for different sound pressure levels, different stack positions and different amplitudes of the temperature gradient. It is therefore possible to draw some conclusions about the physical 
mechanism responsible for the large distorsion of the density (and temperature) fluctuations. In the absence of a temperature gradient and if any source of nonlinearity is discarded, the amplitude of temperature fluctuations are proportional to the acoustic pressure amplitude, but if an axial temperature gradient is applied, the advective term $v^{\prime} d_{x} T_{0}$ of Eq. (1) provides an additional contribution which is proportional to the acoustic velocity and to the temperature gradient. Due to this, the presence of a steep temperature gradient just outside the stack leads to a large increase of temperature fluctuations, which is indeed observed on the fundamental component of $\langle\rho\rangle$ in experiments, and confirmed by theory. As a result, and because the stack end is an important source of nonlinearity, the large amplitude temperature oscillations are significantly distorded, so that both higher harmonics and a time-averaged component are generated locally with a large amplitude.

As mentioned earlier, the linear thermoacoustic theory derived by Rott 4 does not account for several nonlinear effects, and especially for the one investigated in this paper. It would therefore be good to improve available models of thermoacoustic engines (e.g. based on the model proposed here), because the generation of a significant time-averaged component of temperature fluctuations next to the stack termination reveals that some complex mechanisms of heat transport could have an important impact. For instance, the occurrence of a relaxational regime of spontaneous onset/damping of acoustic oscillations has been reported in the present thermoacoustic device [14] (and also in other ones [21]) and some attempts to reproduce such complicated dynamics have failed up to now [15] : the mechanism of temperature distorsion causing nonlinear heat transport next to the heated side of the stack would be (perhaps) a candidate (among others) to explain such kind of experimental observations. More generally, the results presented in this paper are of interest for a more accurate description of thermoacoustic heat transport between the stack/regenerator and the heat exchangers in thermoacoustic (or even in Stirling) engines, a problem which remains an important topical issue for practical applications.

\section{References}

[1] G. W. Swift, Thermoacoustic engines, The Journal of the Acoustical Society of America 84 (4) (1988) 1145-1180.

[2] M. E. H. Tijani, S. Spoelstra, A high performance thermoacoustic engine, Journal of Applied Physics 110 (9) (2011) 093519.

[3] K. Wang, S. R. Sanders, S. Dubey, F. H. Choo, F. Duan, Stirling cycle engines for recovering low and moderate temperature heat : A review, Renewable and Sustainable Energy Reviews 62 (Supplement C) (2016) 89-108.

[4] N. Rott, Thermoacoustics, Advances in Applied Mechanics 20 (1980) 135-175.

[5] S. Boluriaan, P. J. Morris, Acoustic streaming : From rayleigh to today, International Journal of Aeroacoustics 2 (3) (2003) $255-292$.

[6] C. Desjouy, G. Penelet, P. Lotton, J. Blondeau, Measurement of acoustic streaming in a closed-loop traveling wave resonator using laser doppler velocimetry, The Journal of the Acoustical Society of America 126 (5) (2009) 2176-2183.

[7] C. Olivier, G. Penelet, G. Poignand, J. Gilbert, P. Lotton, Weakly nonlinear propagation in thermoacoustic engines : A numerical study of higher harmonics generation up to the appearance of shock waves, Acta Acustica united with Acustica 101 (2015) 941-949.

[8] P. Blanc-Benon, E. Besnoin, O. Knio, Experimental and computational visualization of the flow field in a thermoacoustic stack, Comptes Rendus Mécanique 331 (1) (2003) $17-24$.

[9] A. Berson, G. Poignand, P. Blanc-Benon, G. Comte-Bellot, Nonlinear temperature field near the stack ends of a standingwave thermoacoustic refrigerator, International Journal of Heat and Mass Transfer 54 (21) (2011) 4730-4735.

[10] V. Gusev, P. Lotton, H. Bailliet, S. Job, M. Bruneau, Relaxation-time approximation for analytical evaluation of temperature field in thermoacoustic stack, Journal of Sound and Vibration 235 (5) (2000) 711-726.

[11] K. I. Matveev, G. W. Swift, S. Backhaus, Temperatures near the interface between an ideal heat exchanger and a thermal buffer tube or pulse tube, International journal of heat and mass transfer 49 (5-6) (2006) 868-878.

[12] K. I. Matveev, G. W. Swift, S. Backhaus, Analytical solution for temperature profiles at the ends of thermal buffer tubes, International journal of heat and mass transfer 50 (5-6) (2007) 897-901.

[13] G. Penelet, M. Leclercq, T. Wassereau, P. Picart, Measurement of density fluctuations using digital holographic interferometry in a standing wave thermoacoustic oscillator, Experimental Thermal and Fluid Science 70 (2016) $176-184$.

[14] G. Penelet, M. Guedra, V. Gusev, T. Devaux, Simplified account of rayleigh streaming for the description of nonlinear processes leading to steady state sound in thermoacoustic engines, International Journal of Heat and Mass Transfer 55 (21) (2012) 6042-6053.

[15] M. Guedra, G. Penelet, P. Lotton, Experimental and theoretical study of the dynamics of self-sustained oscillations in a standing wave thermoacoustic engine, Journal of Applied Physics 115 (2) (2014) 024504.

[16] G. Mozurkewich, Time-average temperature distribution in a thermoacoustic stack, The Journal of the Acoustical Society of America 103 (1) (1998) 380-388. 
[17] V. Gusev, P. Lotton, H. Bailliet, S. Job, M. Bruneau, Thermal wave harmonics generation in the hydrodynamical heat transport in thermoacoustics, The Journal of the Acoustical Society of America 109 (1) (2001) 84-90.

[18] A. Berson, P. Blanc-Benon, Nonperiodicity of the flow within the gap of a thermoacoustic couple at high amplitudes, The Journal of the Acoustical Society of America 122 (4) (2007) EL122-EL127.

[19] S. Temkin, Elements of Acoustics, Wiley, 1981.

[20] R. I. Sujith, Transfer matrix of a uniform duct with an axial mean temperature gradient, The Journal of the Acoustical Society of America 100 (4) (1996) 2540-2542.

[21] Z. Yu, A. J. Jaworski, A. S. Abduljalil, Fishbone-like instability in a looped-tube thermoacoustic engine, The Journal of the Acoustical Society of America 128 (4) (2010) EL188-EL194. 